\title{
ANÁLISE ESTATÍSTICA DE INDICADORES DA TUBERCULOSE NO ESTADO DA PARAÍBA
}

\author{
STATISTICAL ANALYSIS INDICATORS OF TUBERCULOSIS IN THE STATE OF PARAÍBA \\ Dalila Camêlo Aguiar ${ }^{\mathrm{a}^{*}}$, Edwirde Luiz Silva Camêlo $^{\mathrm{b}^{* \star}}$, Rayanne Oliveira Carneiro ${ }^{{ }^{* *}}$
}

dalilacamelo@correo.ugr.es ${ }^{\mathrm{a}}$, edwirde@uepb.edu.br ${ }^{\mathrm{b**}}$, rayanneoliveirac@hotmail.com ${ }^{\mathrm{c}^{* *}}$

Universidade de Granada, Espanha*, Universidade Estadual da Paraíba**

\section{RESUMO}

Data de Submissão :28/08/2018

Data de Aceite: 07/10/2018

\begin{abstract}
Objetivo: analisar os principais indicadores epidemiológicos e operacionais da tuberculose nos municípios do Estado da Paraíba no período de 2007 a 2016. Metodologia: Trata-se de um estudo ecológico exploratório, utilizando dados secundários de tuberculose registrados no SINAN. A análise dos dados foi realizada no programa R. Resultados: No período de 2007 a 2016, foram notificados 13.413 casos de tuberculose no estado da Paraíba, com média anual de 1.336,6. A variação da prevalência e incidência de casos novos de tuberculose ao longo dos anos apresenta maiores indicadores entre os anos de 2011 a 2013. Importante declínio ocorreu entre 2013 até 2015, embora em 2015 marque uma nova ascensão no número de casos. As microrregiões de Campina Grande, Cariri Ocidental, Patos e João Pessoa apresentam maiores taxas médias de incidência de tuberculose em suas respectivas mesorregiões. O Cariri Oriental (mesorregião da Borborema) apresenta maior percentual de cura embora Itaporanga (mesorregião do Sertão Paraibano) apresente o menor percentual de cura do estado. Em relação aos pacientes examinados, Curimataú Ocidental exibe maior percentual, no entanto, os menores percentuais são encontrados nas microrregiões do Seridó Oriental e João Pessoa, sendo que esta última lidera nos rankings de incidência média $(>=4,0)$ e prevalência média $(>=4,9)$ de tuberculose por $10 \mathrm{mil} / \mathrm{hab}$. Conclusão: Os resultados apontam a necessidade de reflexão sobre a efetividade das ações de controle e combate à tuberculose no estado visto que a doença estar presente em todos os municípios e apresenta taxas de cura inferiores ao preconizado pela OMS e MS.
\end{abstract}

Palavras-chave: Tuberculose; saúde pública; análise estatística; estado da Paraíba

\section{ABSTRACT}

Objective: To analyze the main epidemiological and operational indicators of tuberculosis in the municipalities of the State of Paraíba in the period 2007-2016. Methodology: It is an exploratory ecological study, using secondary data of tuberculosis registered in the SINAN. Data analysis was performed in program R. Results: In the period studied, 13,413 cases of tuberculosis were reported in the State, with an annual average of 1,336.6. The variation in the prevalence and incidence of new cases of tuberculosis over the years shows higher indicators between the years 2011 to 2013. Important decline occurred between 2013 until 2015, although in 2015 marks a new rise in the number of cases. The microregions of Campina Grande, Cariri Ocidental, Patos and João Pessoa present higher average rates of tuberculosis incidence in their respective mesoregions. O Cariri Oriental presents a higher percentage of cure, although Itaporanga presents the lowest percentage of cure for the state. In relation to the patients examined, Curimataú Ocidental presents a higher percentage; however, the lowest percentages are found in the microregions of Seridó Oriental and João Pessoa, the latter being that the latter leads the rankings of average incidence $(>=4.0)$ and mean prevalence $(>=4.9)$ of tuberculosis 10 thousand/inhab. Conclusion: The results point to the need for reflection on the effectiveness of control actions and combat tuberculosis in the state since the disease is present in all municipalities and has rates of cure lower than that recommended by the WHO and the Brazilian Ministry of Health.

Keywords: Tuberculosis; public health; statistical analysis; state of Paraíba 


\section{Introdução}

A tuberculose é uma doença infectocontagiosa causada pelo Mycobacterium Tuberculosis. Também denominada Bacilo de Koch, a bactéria foi identificada em 1882 pelo pesquisador e bacteriologista alemão Robert Koch (18431910), sendo este evento um marco no avanço do conhecimento da doença, que tem sua cadeia epidemiológica e tratamento conhecidos há longo tempo, porém persiste como um considerável problema de saúde pública em muitos países. Entre as doenças negligenciadas, a tuberculose atinge os mais pobres, os vulneráveis, os marginalizados, possuindo uma estreita relação com as situações de subnutrição, condições precárias de habitação e baixo acesso aos serviços de saúde, quem durante séculos têm carregado o maior fardo da doença ${ }^{1-4}$.

A transmissão da tuberculose acontece de pessoa a pessoa por via aérea a partir da inalação de aerossóis, produzidos pela tosse, espirro ou fala de doentes com tuberculose ativa nas formas pulmonar e laríngea. Casos em que é possível visualizar no exame de microscopia (baciloscopia de escarro) a presença de bacilos diretamente no escarro (casos bacilíferos), são os responsáveis pela manutenção da cadeia de transmissão. Dessa forma, descarta-se a transmissão por compartilhamento de alimentos, objetos, por apertos de mão ou por qualquer outra via que não exponha o indivíduo aos aerossóis expelidos pelo doente ${ }^{5-6}$.

Enquanto o indivíduo estiver eliminando bacilos no escarro a transmissão ainda acontece, no entanto, uma vez dado início ao esquema terapêutico adequado, a transmissão tende a diminuir gradativamente, podendo-se perceber que, no fim do primeiro mês de tratamento adequado, o paciente já apresenta uma atenuação dos sintomas e, decorrido o segundo mês, o doente já está praticamente assintomático e ganhando peso. Caso isso não aconteça pode ser sugestivo de erro de diagnóstico, associação com outra doença, como a neoplasia de pulmão, ou resistência aos medicamentos em uso ${ }^{5,7}$.

A tuberculose é uma doença caracterizada nacional e mundialmente como um problema de saúde pública muito embora possua cura na maioria dos casos diante de um diagnóstico oportuno e um tratamento efetivo, porém, diante dos índices de casos novos e de morbimortalidade a doença requer um olhar voltado à prevenção, combate e tratamento.

A Organização Mundial de Saúde (OMS) aponta que, no mundo, 10,4 milhões de pessoas tiveram tuberculose em 2015, e mais de 1 milhão morreram por conta da doença, classificando em três listas, os 30 países prioritários no controle da tuberculose para o período de 2016 a 2020, somando-se 48 países prioritários para a abordagem da doença ${ }^{8}$. Tal situação colocou o Brasil na $20^{\text {a }}$ posição na classificação de carga da doença9 ${ }^{9}$. No ano de 2013, o país notificou no Sistema de Notificação de Agravos de Notificação (SINAN $)^{10} 88.450$ casos, a Paraíba apresentou três casos a mais que em 2012 (1.429), sendo 152 no município de Campina Grande, dividindo-se em casos pulmonares, extrapulmonares e pulmonar e extrapulmonar concomitantemente ${ }^{11}$.

Já a taxa de mortalidade em 2014 foi de 1,7\% ficando no mesmo patamar do ano de 1990 abaixo da média do Nordeste, de 2,5\% do total de casos notificados. Em 2014 a cada 100.000 habitantes, a Paraíba ocupava a $18^{\mathrm{a}}$ posição no ranking nacional no coeficiente de incidência $(24,5)$ e 1,7 para o coeficiente de mortalidade ${ }^{12,13}$.

Em 2015, foram 10,4 milhões de novos casos de tuberculose, nos últimos cinco anos, a infecção tem sido líder como causa de morte de um único agente infeccioso, classificação acima HIV/AIDS. No Brasil, em 2016, foram diagnosticados e registrados 66.796 casos novos e 12.809 casos de retratamento de tuberculose $\mathrm{e}^{14,15}$.

O Brasil é um país caracterizado por suas grandes dimensões e diferenças de acordo com cada região, a tuberculose por sua vez, possui incidência e perfis diferentes conforme a região considerada, porém, em todas as regiões a doença é considerada um problema de saúde pública. Em regiões cujas condições socioeconômicas são precárias, a tuberculose mostra-se mais incidente, principalmente por características como pobreza, déficit educacional, desnutrição e aglomerados populacionais ${ }^{16}$.

Tendo em vista o perfil da Tuberculose no cenário mundial, brasileiro e paraibano, torna-se evidente a importância da realização de estudos 
que analisem o comportamento da doença nestas esferas. O presente estudo tem como objetivo descrever principais indicadores epidemiológicos e operacionais da tuberculose nas microrregiões e mesorregiões do Estado da Paraíba, considerando a evolução temporal dos casos, no período compreendido entre 2007 e 2016.

\section{Materiais e métodos}

Esta pesquisa é um estudo ecológico exploratório da evolução temporal dos indicadores da tuberculose com base nos casos notificados no Estado da Paraíba registrados no SINAN e disponibilizados no site do Departamento de Informática do Sistema Único de Saúde (DATASUS $)^{10}$. Como unidades de análise, foram utilizadas as microrregiões e mesorregiões do estado, caracterizando-se como propósito secundário, a sua descrição espacial.

A Paraíba é uma das 27 unidades federativas do Brasil, localizada na região leste do Nordeste. Constituído de 223 municípios, 4 mesorregiões e 23 microrregiões. Segundo o Censo 2010 realizado pelo (IBGE) ${ }^{17}$, o estado conta com uma população de 3.766 .528 habitantes, sendo que 2.838 .678 residem na zona urbana e 927.850 na zona rural.

As variáveis incluídas para análise neste estudo foram os indicadores epidemiológicos (incidência e prevalência) e indicadores operacionais (proporção de cura, proporção de não-cura e proporção de pacientes examinados). Para análise dos dados, foi utilizado o programa $\mathrm{R}^{18}$ versão 3.4.3.

Por se tratar de uma pesquisa de dados secundários e não envolver diretamente seres humanos, este estudo não foi submetido à avaliação do Comitê de Ética em Pesquisa.

\section{Resultados e Discussão}

No período de 2007 a 2016, foram notificados 13.413 casos de tuberculose no estado da Paraíba, com média de 1.336,6 casos anualmente, sendo que
$68,3 \%$ dos casos eram homens e $31,7 \%$ mulheres. Os indicadores epidemiológicos de prevalência e incidência para o período são mostrados na Figura 1.

Figura 1 - Distribuição do coeficiente de incidência e prevalência dos casos de tuberculose/100.000 habitantes no Estado do Paraíba entre os anos de 2007 a 2016.

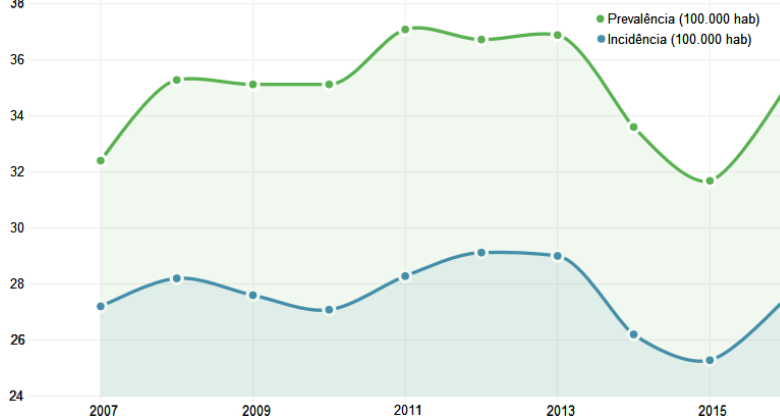

Fonte: Elaborado pelos autores, 2018

A variação da prevalência e incidência ao longo dos anos mostra o desempenho da Paraíba quanto ao índice de casos novos de tuberculose diagnosticados que, entre os anos estudados, percebe-se maiores indicadores entre os anos de 2011 a 2013, conforme também mostra a literatura relacionada ao número de casos diagnosticados e registrados no banco de dados do SINAN/PB. Os registros apresentam um importante declínio até o ano de 2015, ano este que marca uma nova ascensão no número de casos.

A Figura 2 apresenta a incidência média de tuberculose nas microrregiões por mesorregião. 
Figura 2 (a) - Incidência média de tuberculose nas mesorregiões da Paraíba por 10 mil/hab., 2007-2016.

\section{Mesorregiao}

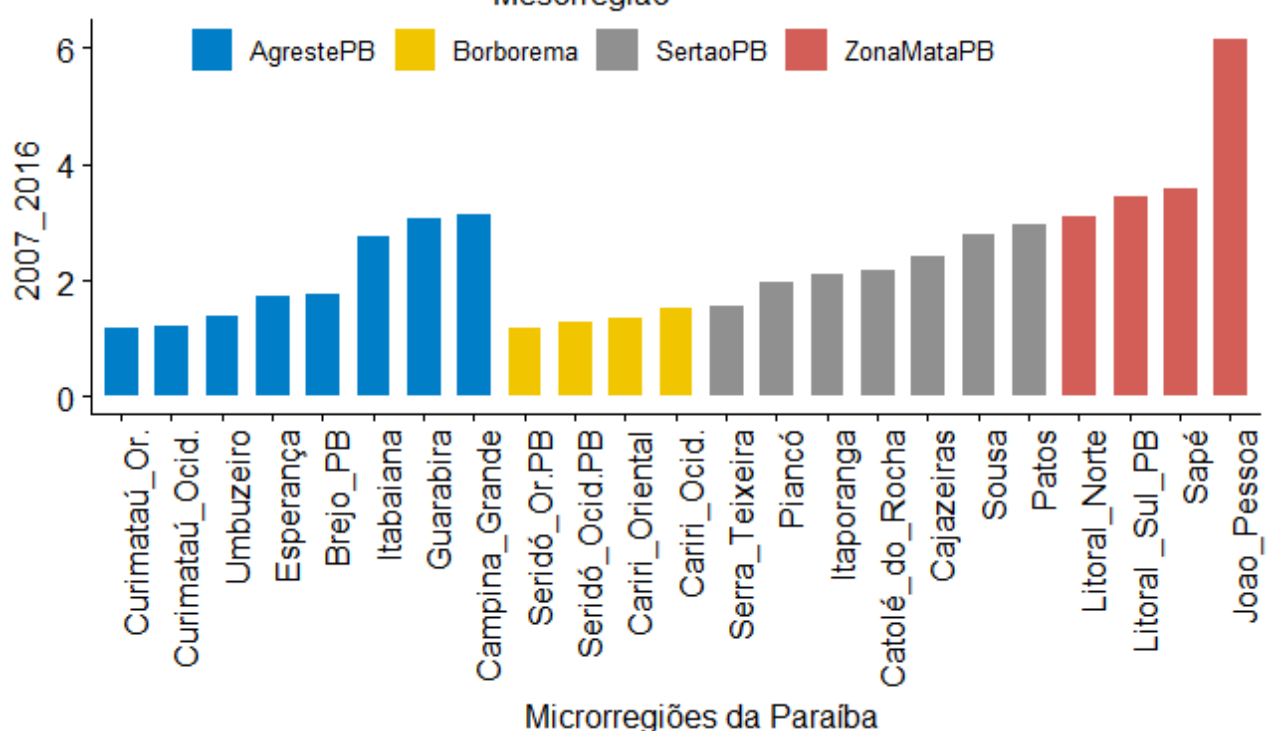

Fonte: Elaborado pelos autores, 2018.

As microrregiões de Campina Grande, Cariri Ocidental, Patos e João Pessoa lideram as maiores taxas médias de incidência de tuberculose em suas respectivas mesorregiões, respectivamente as menores são Curimataú Oriental, Seridó Oriental Paraibano, Serra de Teixeira e Litoral Norte.
A Figura 2 (b) apresenta o mesmo resultado anterior, no entanto de forma decrescente, permitindo melhor visualização da incidência média de tuberculose nas microrregiões por mesorregião.

Figura 2 (b): Incidência média de tuberculose nas mesorregiões da Paraíba por 10 mil/hab., 2007-2016.

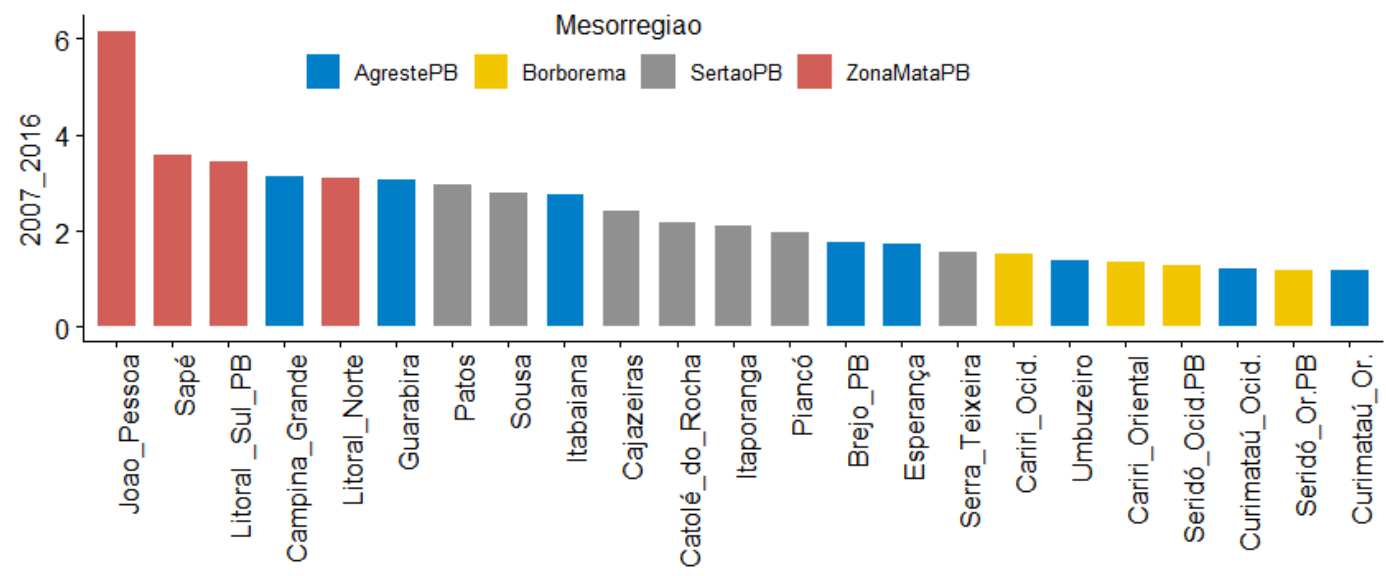

Microrregiões da Paraiba

Fonte: Elaborado pelos autores, 2018.

Percebe-se que as cinco primeiras microrregiões representam as maiores taxas médias de tuberculose, quatro delas compõe a mesorregião da Zona da Mata Paraibana, exceto a microrregião de Campina Grande que pertence ao Agreste Paraibano. Em contrapartida, as microrregiões da Borborema apresentam as menores taxas médias, embora a microrregião do Curimataú Oriental situada no Agreste Paraibano apresente a menor taxa.

A Figura 3 apresenta a variação dos indicadores "cura", "não-cura" e "casos examinados". 
Figura 3 - Variação percentual dos índices de cura, não-cura e pacientes examinados com tuberculose na Paraíba, 20072016

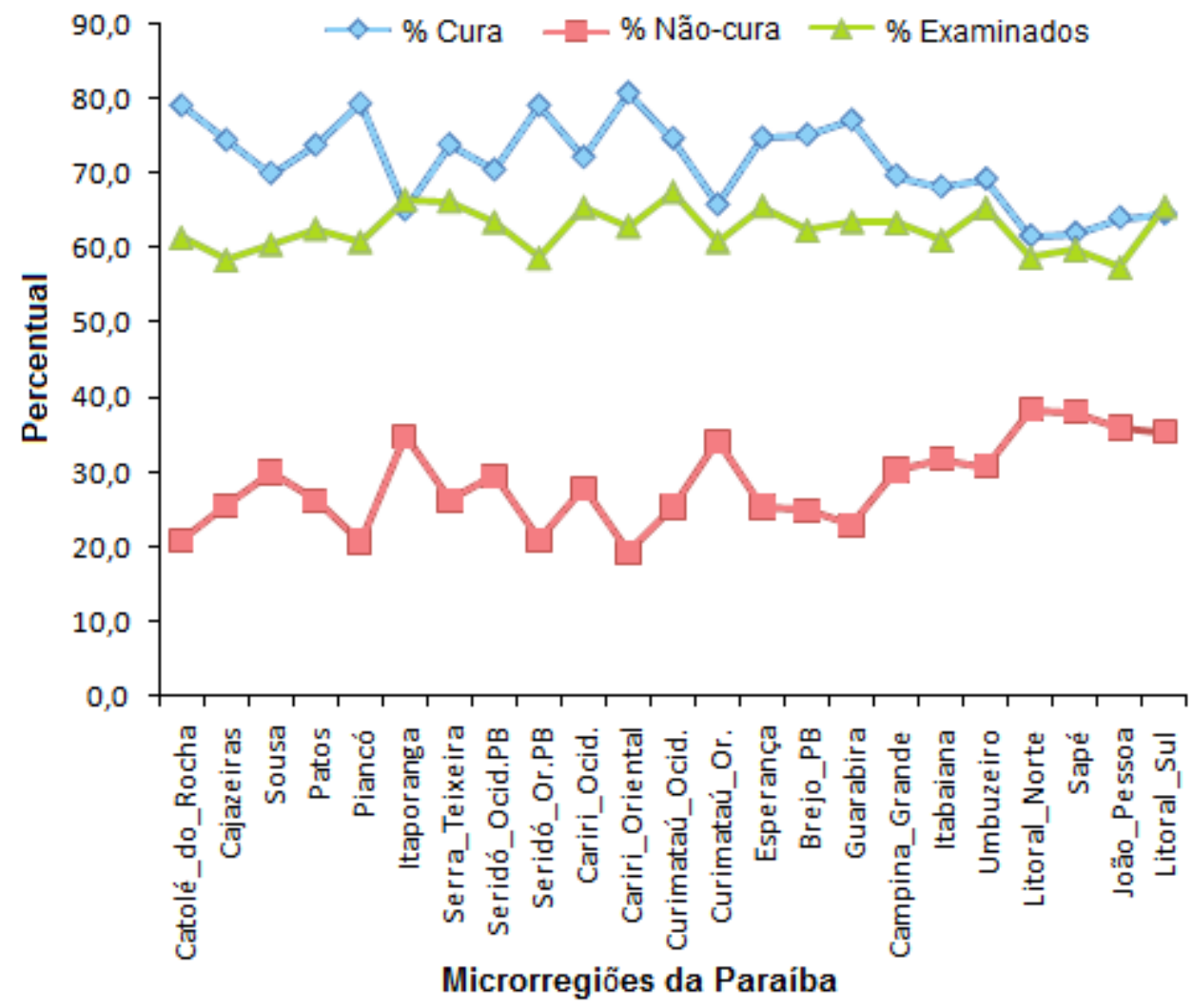

Fonte: Elaborado pelos autores, 2018.

Observa-se índices de cura inferiores ao preconizado pela Organização Mundial da Saúde e Ministério da Saúde. Estas esferas preconizam que, taxas de cura inferiores a $85 \%$ dos casos de tuberculose diagnosticados e tratados, revelam a importância do uso de estratégias que visem à adesão ao tratamento, diminuição do abandono e, consequentemente das taxas de não-cura e prevenção de cepas resistentes aos tuberculostáticos, a exemplo da implementação da estratégia de Tratamento Diretamente Observado (TDO), o que já é realizado no estado da Paraíba. A microrregião do Cariri Oriental apresenta maior percentual de cura e as microrregiões de Itaporanga e Curimataú Oriental, repectivamente apresentam os menores percentuais. Em relação aos pacientes examinados, Curimataú Ocidental apresenta maior percentual, no entanto, os menores percentuais são encontrados nas microrregiões do Seridó Oriental e João Pessoa.
A mesorregião de João Pessoa (Litoral Norte, Sapé, João Pessoa e Litoral Sul) apresenta maior taxa média de incidência, muito embora seja uma área prioritária de atenção à doença e possua uma rede de atendimento aos doentes de tuberculose que disponibilizam diagnóstico oportuno e tratamento efetivo anti-tuberculose. É necessário destacar que situado na microrregião de João Pessoa, o município de João Pessoa é o mais populoso do estado da Paraíba e para onde muitos casos são encaminhados para investigação da sintomatologia, realização do diagnóstico e início do esquema terapêutico, muitas vezes mantendo pacientes com quadro clínico mais complexo internados em unidades hospitalares até que seja possível dar continuidade ao uso de drogas tuberculostáticas em seu município.

A Figura 4 apresenta a distribuição espacial dos coeficientes médios de incidência. 
Figura 4 - Distribuição espacial dos coeficientes médios de incidência de casos novos de tuberculose/10.000 habitantes nas microrregiões do Estado da Paraíba entre os anos de 2007 a 2016.

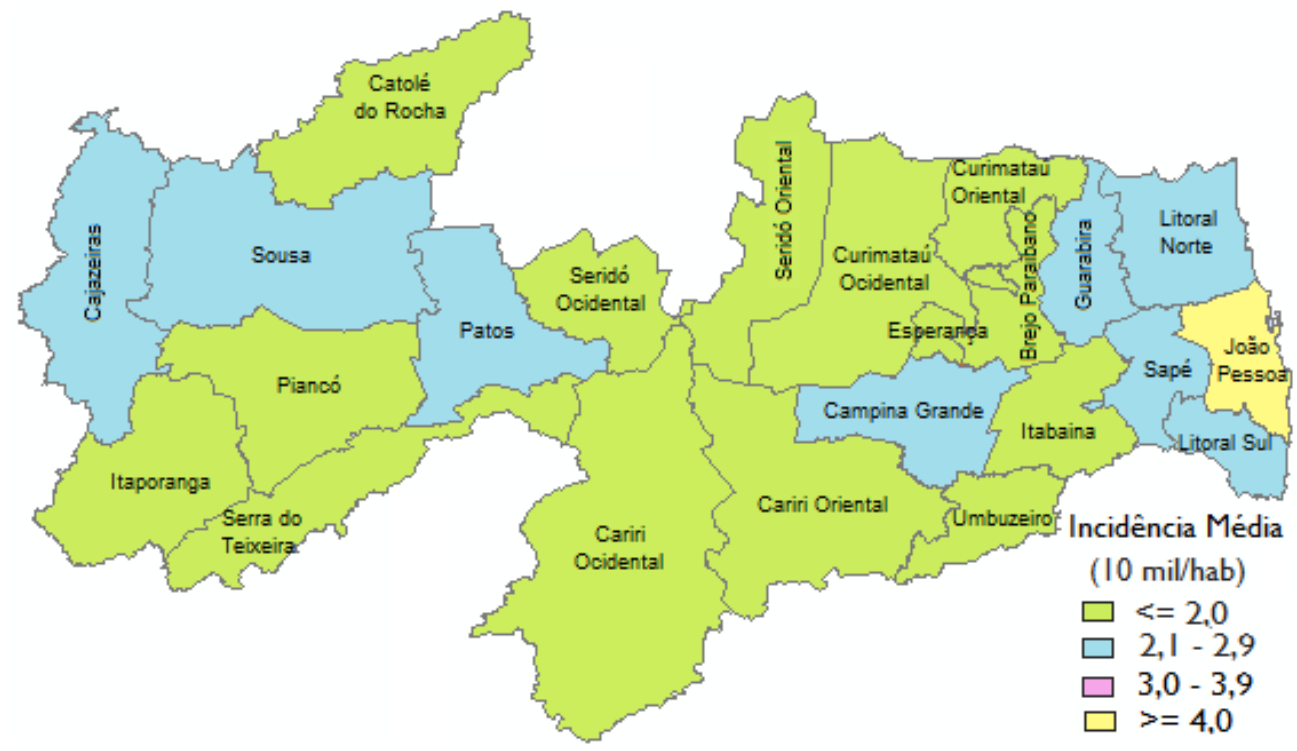

Fonte: Elaborado pelos autores, 2018.

Percebe-se que 60,9\% (14) das microrregiões exibem taxas de incidência média por $10 \mathrm{mil} /$ hab inferior ou igual 2,0, em contrapartida a microrregião de João Pessoa (cor amarela) apresenta maior incidência média $(>=4,0)$ de tuberculose por $10 \mathrm{mil} / \mathrm{hab}$.
A Figura 5 apresenta a distribuição espacial dos coeficientes médios de prevalência da tuberculose nas microrregiões do estado da Paraíba.

Figura 5 - Distribuição espacial dos coeficientes médios de prevalência de casos novos de tuberculose/10.000

habitantes nas microrregiões do Estado da Paraíba entre os anos de 2007 a 2016.

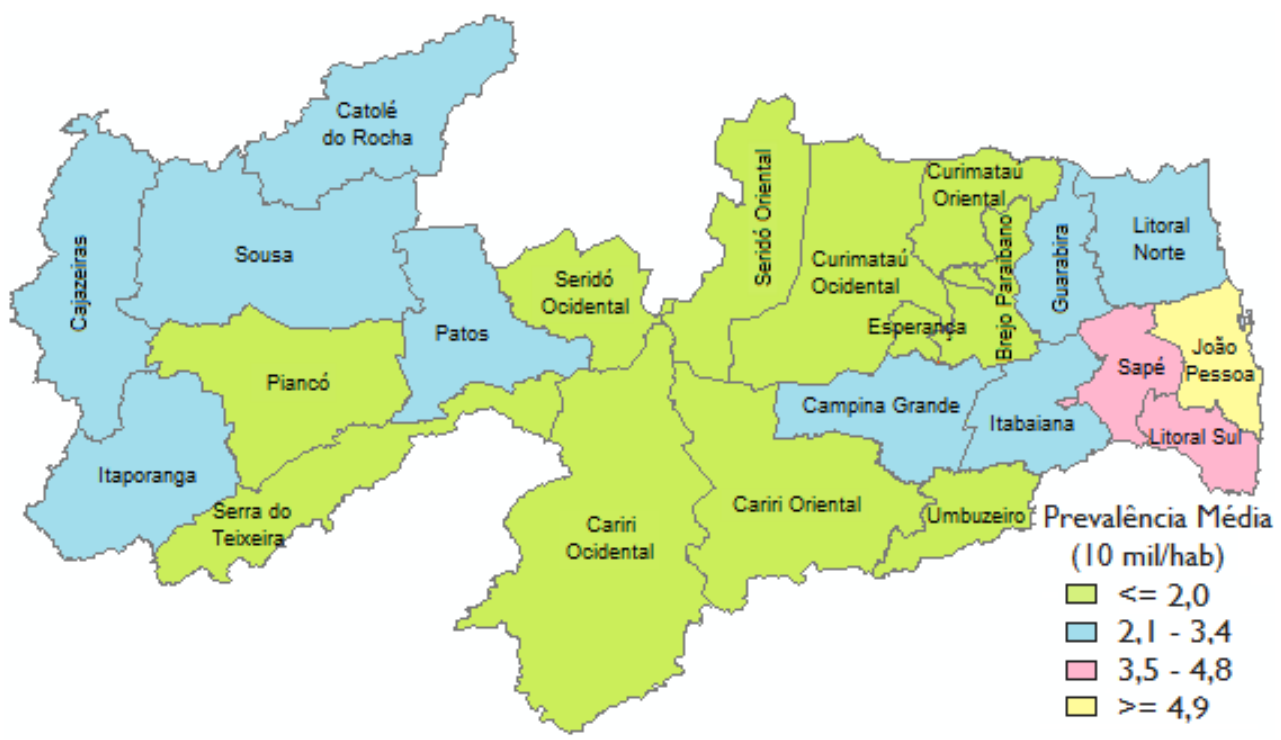

Fonte: Elaborado pelos autores, 2018. 
Percebe-se que onze $(47,8 \%)$ das microrregiões exibem taxas de prevalência média por $10 \mathrm{mil} / \mathrm{hab}$ igual ou inferior a 2,0 , nove $(39,1 \%)$ entre 2,1-3,4, Litoral Sul e Sapé (3,54,8 ), e por último, a microrregião de João Pessoa (cor amarela) lidera o ranking no estado com prevalência média $(>=4,9)$ de tuberculose por 10 $\mathrm{mil} / \mathrm{hab}$.

\section{Conclusão}

A tuberculose na Paraíba, tem se mostrado uma doença presente em todo o território, destacando-se principalmente as microrregiões da mesorregião da Zona da Mata quanto aos indicadores da tuberculose e nos levando à reflexão da efetividade das ações de controle e combate à doença realizada no estado, embora em algumas localidades o índice de casos novos tenha se mostrado inferiores quando comparadas às demais, as taxas de cura inferiores a $85 \%$ confirmam a importância de buscar-se por estratégias ainda mais eficientes.

A realização de estudos como este que permitem compreender a apresentação da doença de forma que seja possível analisar sua distribuição em localidades específicas é de suma importância para a saúde coletiva, ao passo que proporciona a compreensão das localidades mais afetadas e, consequentemente, alerta para a necessidade de ações de saúde mais efetivas.

\section{Referências}

1. Lopes RH, Menezes RMP, Costa TD, Queiroz AAR, Cirino ID, Garcia MCC. Fatores associados ao abandono do tratamento da tuberculose pulmonar: uma revisão integrativa. Revista Baiana de Saúde Pública 2014; 37 (3): 661-671

2. Ethel M, Noia L. Estratégias da agenda pós-2015 para o controle da tuberculose no Brasil: desafios e oportunidades. Epidemiologia e Serviços de Saúde 2016; 25 (2): 423-426.

3. Pedro HSP, Nardi SMT, Finardi AJ, Moraes EB, Oliveira RS, Pereira MIF, Machado RLD, Castiglioni L. Cenário atual da tuberculose. Hansen Int. 2014; 39 (1): 40-55.

4. Rêgo LP, da Cunha FF, Rodrigues ILA, Nogueira LMV. Assistência humanizada de enfermagem às pessoas doentes com tuberculose: revisão integrativa $2002-2012$ Revista Baiana de Saúde Pública 2015; 38 (3): 738-750.

5. Brasil. Ministério da Saúde. Secretaria de Vigilância em Saúde. Guia de Vigilância em Saúde. Brasília, DF: Ministério da Saúde; 2014.

6. Winter, BCA, Carollo B, Garrido RG. A tuberculose no cárcere: um retrato das mazelas do sistema prisional brasileiro. Medicina Legal de Costa Rica 2017; 34 (2): 1-12.

7. Siqueira HR. Enfoque Clínico da Tuberculose Pulmonar. Pulmão RJ 2012; 21 (1): 15-18.

8. World Health Organization (WHO). Global tuberculosis control: surveillance, planning, financing. Geneva, 2016.

9. Brasil. Ministério da Saúde. Secretaria de Vigilância em Saúde. Perspectivas brasileiras para o fim da tuberculose como problema de saúde pública. Boletim Epidemiológico 2016; 47 (13): 1-15.

10. Brasil. Ministério da Saúde. Sistema de Informação de Agravos de Notificação Tuberculose - casos confirmados no Sistema de Informação de Agravos de Notificação SINAN. Brasília, DF; 2017 [citado em 2018 junho 7]. Disponível em: http://www2.datasus.gov.br/.

11. Barros PG, Pinto ML, Silva TC, Silva EL, Figueiredo, TMRM. Perfil Epidemiológico dos casos de Tuberculose Extrapulmonar em um município do estado da Paraíba, 2001-2010. Cad. saúde colet. [online] 2014; 22 (4): 343 350. doi: 10.1590/1414-462X201400040007.

12. Brasil. Ministério da Saúde. Fundação nacional de Saúde. Controle da Tuberculose: uma proposta de integração ensino-serviço. Brasília: Ministério da Saúde; 2002.

13. Carneiro WS, Evangelista BP, Sousa ARQ, Cavalcante MKS, Nogueira, JA. Espacialização dos casos de tuberculose na Paraíba. I Congresso Nacional de Ciências da Saúde - CONACIS. Cajazeiras: Editora Realize, 2014.

14. Brasil. Ministério da Saúde. Secretaria de Vigilância em Saúde. Indicadores prioritários para o monitoramento do Plano Nacional pelo Fim da Tuberculose como Problema de Saúde Pública no Brasil. Boletim Epidemiológico 2017; 48 (8): 1-11.

15. World Health Organization (WHO). Global tuberculosis control: surveillance, planning, financing. Geneva, 2017.

16. Coutinho, LASA, Oliveira DS, Souza GF, Fernandes Filho GMC, Saraiva MG. Perfil Epidemiológico da Tuberculose no Município de João Pessoa - PB, entre 2007 - 2010 Revista Brasileira de Ciências da Saúde 2012; 16 (1): 35 42. doi: 10.4034/RBCS.2012.16.01.06.

17. Censo Demográfico 2010. Características da população e dos domicílios: resultados do universo. Rio de Janeiro: IBGE, 2011 [citado em 2018 maio 7]. Disponível em: https://ww2.ibge.gov.br/home/estatistica/populacao/ censo2010/ 
18. $\mathrm{R}$ Core Team. R: A language and environment for statistical computing. $\mathrm{R}$ Foundation for Statistical Computing, Vienna, Austria. 2017. url: https://www.Rproject.org/.

\section{Como citar este artigo:}

Aguiar DC, Camêlo ELS, Carneiro RO. Análise estatística de indicadores da tuberculose no estado da Paraíba. Rev. Aten. Saúde. 2019; 17(61): 05-12. 